\title{
Facile heterocyclic synthesis and antimicrobial activity of polysubstituted and condensed pyrazolopyranopyrimidine and pyrazolopyranotriazine derivatives
}

\author{
HEND N. HAFEZ ${ }^{1,2}$ \\ ABDULRAHMAN G. ALSHAMMARI ${ }^{1}$ \\ ABDEL-RHMAN B. A. EL-GAZZAR ${ }^{1,2}$ \\ ${ }^{1}$ Al-Imam Mohammad Ibn Saud \\ Islamic University (IMSIU) \\ Faculty of Science \\ Department of Chemistry \\ P.O. Box 90950 Riyadh 11623 \\ Kingdom of Saudi Arabia \\ ${ }^{2}$ Photochemistry Department \\ (Heterocyclic and Nucleosides Unit) \\ National Research Centre \\ Cairo, Egypt
}

Accepted December 22, 2014

\begin{abstract}
Reaction of 6-amino-3-methyl-4-(substituted phenyl)-1,4dihydropyrano[2,3-c]pyrazole-5-carbonitrile (1) with triethylorthoformate followed by treatment with hydrazine hydrate, formic acid, acetic acid, phenylisocyanate, ammonium thiocyanate and formamide afforded the corresponding pyranopyrimidine derivatives 2-6. Cyclocondensation of 1 with cyclohexanone afforded pyrazolopyranoquinoline 7. One-pot process of diazotation and de-diazochlorination of 1 afforded pyrazolopyranotriazine derivative 8, which upon treatment with secondary amines afforded 9 and $10 a-$ c. Condensation of $\mathbf{2}$ with aromatic aldehyde gave the corresponding Schiff bases $\mathbf{1 1} \mathbf{a}, \mathbf{b}$, the oxidative cyclization of the hydrazone with appropriate oxidant afforded 11-(4-fluorophenyl))-2-(4-substituted phenyl)-10-methyl-8,11-dihydropyrazolo- $\left[4^{\prime}, 3^{\prime}: 5,6\right]$ pyrano[3,2-e][1,2,4]triazolo[1,5-c]pyrimidines $(\mathbf{1 2} \mathbf{a}, \mathbf{b})$. Structures of the synthesized compounds were confirmed by spectral data and elemental analysis. All synthesized compounds were evaluated for antibacterial and antifungal activities compared to norfloxacin and fluconazole as standard drugs. Compounds 9, 10c, 12a and 15 were found to be the most potent antibacterial agents, with activity equal to that of norfloxacin. On the other hand, compound 5 exhibited higher antifungal activity compared to fluconazole.
\end{abstract}

Keywords: pyrazolopyranopyrimidine, pyrazolopyranotriazine, pyrazolopyranoquinoline, antimicrobial activity

Antibiotic resistance, which results from inappropriate and irrational use of antimicrobial medicines, provides favorable conditions for resistant bacteria to emerge. This resistance of pathogenic bacteria towards available antibiotics is rapidly becoming a major worldwide problem. Hence, the design of new compounds to deal with resistant bacteria has become one of the most important goals of antibacterial research today.

\footnotetext{
*Correspondence; e-mail: profelgazzar@yahoo.com
} 
H. N. Hafez et al.: Facile heterocyclic synthesis and antimicrobial activity of polysubstituted and condensed pyrazolopyranopyrimidine and pyrazolopyranotriazine derivatives, Acta Pharm. 65 (2015) 399-412.

In continuation of our study of the chemistry of heterocyclic $\beta$-enamino-carbonitrile (1) and the synthesis of fused pyrane ring systems of pharmacological importance (2), pyrane and its fused derivatives have attracted great interest owing to their antimicrobial (3-5), antiviral (6), antitumor (7), antiproliferactive (8), molluscicidal (9), and anti-inflammatory activities (10). Also, pyrane derivatives are well known antihistaminic agents (11). Moreover, it has been noticed that introduction of an additional ring to the pyrimidine core tends to exert a profound influence in conferring novel biological activities in these molecules (12-14). Pyrimidine and their fused derivatives play an essential role in several biological processes and have considerable chemical and pharmacological importance. In particular, pyrimidine nucleus can be found in a broad variety of antibacterial and antitumor agents, as well as in agrochemical and veterinary products $(15,16)$. Some substituted pyrano[2,3-c]pyrazoles have been found to be effective antiplatelet molecules (17), which effect $\mathrm{K}^{+}$-induced calcium-dependent aortal contraction. Several pyrano[2,3-c]-pyrazol-4ones have demonstrated affinity toward A1 and A2a adenosine receptors (18). Also, 6-amino-5-cyano-dihydro-pyrano[2,3-c]pyrazoles have been identified as a screening hit for human Chk1 kinase inhibitors (19). We herein report the use of 6-amino-3-methyl-4-(4florophenyl)-1,4-dihydropyrano[2,3-c]pyrazole-5-carbonitrile (1) as starting material for the synthesis of a new series of pyrazolopyranopyrimidines (2-6) and their derivatives $\mathbf{1 1 a}, \mathbf{b}$, pyrazolopyranoquinoline (7) and pyrazolopyranotriazine (8).

\section{EXPERIMENTAL}

All melting points were measured on an Electrothermal 9100 series digital melting point apparatus (Shimadzu, Japan). Microanalytical data were provided by a Vario Elementar apparatus (Shimadzu, Japan). Elemental analyses of all compounds were within \pm $0.4 \%$ of the theoretical values. Physicochemical data are given in Table I. The IR spectra (KBr) were recorded on a Perkin Elmer 1650 spectrometer (USA). ${ }^{1} \mathrm{H}$ spectra were recorded on a JEOL EX-300 and JEOL ECA-500 (Japan). Chemical shifts were expressed in ppm relative to $\mathrm{SiMe}_{4}$ as internal standard in DMSO- $d_{6}$ as a solvent. Mass spectra were recorded on a $70 \mathrm{eV}$ Finnigan SSQ 7000 spectrometer (Thermo-Instrument System Incorporation, USA) (Table II). The purity of the compounds was checked on aluminium plates coated with silica gel (Merck, Germany). Chemicals and solvents were purchased from Sigma-Aldrich (USA). Norfloxacin and fluconazole were supplied by Pasteur laboratory (Giza, Egypt). Compound 1 was synthesized according to the reported procedure (20).

\section{Syntheses}

6-Amino-3-methyl-4-fluorophenyl-1,4-dihydro-pyrano[2,3-c]pyrazole-5-carbonitrile (1). Ethyl-3-(2-carbamo-thioylhydrazineylidene)-butanoate $(2.03 \mathrm{~g}, 10 \mathrm{mmol})$ was added into sodium ethoxide solution $(20 \mathrm{~mL})$ followed by 2-(4-flurobenzylidene)-malononitrile $(1.71 \mathrm{~g}$, $10 \mathrm{mmol}$ ). The reaction mixture was heated under reflux for $6 \mathrm{~h}$. The compound obtained was crystallized as yellow powder.

4-(4-Fluorophenyl)-5-imino-3-methyl-1,4-dihydropyrazolo[4',3':5, 6]pyrano[2,3-d]pyrimidin-6(5H)-amine (2). - Compound $1(0.01 \mathrm{~mol})$ was added to a mixture of triethylorthoformate $(0.01 \mathrm{~mol})$ and acetic anhydride $(20 \mathrm{~mL})$, and the reaction mixture was refluxed for 
H. N. Hafez et al.: Facile heterocyclic synthesis and antimicrobial activity of polysubstituted and condensed pyrazolopyranopyrimidine and pyrazolopyranotriazine derivatives, Acta Pharm. 65 (2015) 399-412.

$5 \mathrm{~h}$. The solvent was removed under reduced pressure. The separated solid was refluxed with hydrazine hydrate $(0.01 \mathrm{~mol})$ in absolute ethanol $(50 \mathrm{~mL})$. The reaction mixture was refluxed for $2 \mathrm{~h}$, concentrated, cooled, and the solid product that separated out was filtered off and recrystallized as yellow powder.

4-(4-Substituted phenyl)-3-methyl-4,8-dihydropyrazolo[4',3':5,6]pyrano[2,3-d]pyrimidine $5(1 \mathrm{H})$-ones $(3 \boldsymbol{a}, \boldsymbol{b})$. General procedure. - A mixture of $\mathbf{1}(0.01 \mathrm{~mol})$ and appropriate acid (formic and/or acetic acid) was refluxed. The solvent was removed under reduced pressure and the separated solid was recrystallized from appropriate solvent in a good yield to give $\mathbf{3 a}, \mathbf{b}$ respectively.

N-[4-(4-fluorophenyl)-3-methyl-7-oxo-6-phenyl-4,6,7,8-tetrahydropyrazolo[4',3':5,6]pyrano[2,3-d]pyrimidine-5(1H)-ylidene]benzamide $(4)$. - Phenyl isocyanate $(0.01 \mathrm{~mol})$ and triethylamine $(0.5 \mathrm{~mL})$ were added to a solution of $\mathbf{1}(0.01 \mathrm{~mol})$ in ethanol $(20 \mathrm{~mL})$. The reaction mixture was refluxed for $3 \mathrm{~h}$, cooled and the resulting solid was filtered off and recrystallized to give 4 as yellow powder.

N-[4-(4-fluorophenyl)-3-methyl-7-thioxo-1,4,7,8-tetrahydropyrazolo[4',3':5,6]pyrano[2,3-d]pyrimidine-5-yl]thiourea (5). - Ammonium thiocyanate $(0.03 \mathrm{~mol})$ was added to a solution of $1(0.01 \mathrm{~mol})$ in acetic acid $(15 \mathrm{~mL})$ and the reaction mixture was refluxed for $10 \mathrm{~h}$. The solid that separated upon cooling and dilution with water was filtered off and purified to give 5 as pale brown powder.

4-(4-Fluorophenyl)-3-methyl-1,4-dihydropyrazolo[4',3':5,6]pyrano[2,3-d]pyrimidin5-amine (6). - Compound $1(0.01 \mathrm{~mol})$ was added to a mixture of formamide $(10 \mathrm{~mL})$, formic acid (5 $\mathrm{mL}$ ) and dimethylformamide $(5 \mathrm{~mL})$. The reaction mixture was refluxed for $12 \mathrm{~h}$. The solid that separated on cooling was filtered off as pale yellow powder.

4-(4-Fluorophenyl)-3-methyl-1,4,6,7,8,9-hexahydropyrazolo[4',3':5,6]pyrano[2,3-b]quinolin5-amine (7). - Cyclohexanone ( $2.8 \mathrm{mmol})$ was added to a solution of $\mathbf{1}(2 \mathrm{mmol})$ in a mixture of dichloroethane/THF (2:1). After stirring for $5 \mathrm{~min}$ at room temperature, aluminum chloride $(4 \mathrm{mmol})$ was added, and the mixture was heated under reflux for $2 \mathrm{~h}$. The mixture was cooled to room temperature and aluminum chloride $(4 \mathrm{mmol})$ was added. The mixture was refluxed $2 \mathrm{~h}$. The solvent was removed under reduced pressure. An aqueous solution of sodium hydroxide $(50 \mathrm{~mL}, 10 \%)$ was added. After stirring for $30 \mathrm{~min}$, the precipitate was filtered, washed twice with $25 \mathrm{~mL}$ of water and with $10 \mathrm{~mL}$ of ether, dried at room temperature until constant mass, purified in $5 \mathrm{~mL}$ of acetonitrile, and filtered while hot as white powder.

4-Chloro-5-(4-fluorophenyl)-6-methyl-5,8-dihydro-pyrazolo[4',3':5,6]pyrano[2,3-d] [1,2,3]-triazine (8). - A solution of sodium nitrite $(11.4 \mathrm{mmol})$ in water $(7 \mathrm{~mL})$ was added for $15 \mathrm{~min}$ to a suspension of the foregoing compound $1(8.1 \mathrm{mmol})$ at $0-5{ }^{\circ} \mathrm{C}$ in concentrated hydrochloric acid $(16 \mathrm{~mL})$. The resulting mixture was stirred at $0{ }^{\circ} \mathrm{C}$ for further $40 \mathrm{~min}$ and then allowed to stand at room temperature overnight. The reaction mixture was quenched in water $(100 \mathrm{~mL})$. The precipitate was washed twice with $15 \mathrm{~mL}$ of water and dried under room temperature, purified in $2 \mathrm{~mL}$ of acetonitrile, and filtered while hot to give 8 as orange powder.

5-(4-Fluorophenyl)-4-(pyrrolidine, morpholine, piperazine, and/or N-(methyl-piperazinyl)-6methyl-5,8-dihydropyrazolo[4',3':5,6]pyrano[2,3-d][1,2,3]triazines $(\mathbf{9}, \mathbf{1 0} \boldsymbol{a}-\boldsymbol{c})$. General procedure. - Compound 8 (1 mmol) and the corresponding amine (5 mmol) were fused in a sand bath for $6 \mathrm{~h}$. The mixture was cooled at room temperature and poured into ethanol $(30 \mathrm{~mL})$ 
H. N. Hafez et al.: Facile heterocyclic synthesis and antimicrobial activity of polysubstituted and condensed pyrazolopyranopyrimidine and pyrazolopyranotriazine derivatives, Acta Pharm. 65 (2015) 399-412.

under stirring. When a precipitate was formed, it was filtered off, washed twice with 15 $\mathrm{mL}$ of ethanol and twice with $8 \mathrm{~mL}$ of diethyl ether, and then dried at room temperature overnight.

5-(4-Fluorophenyl)-6-methyl-4-pyrrolidin-1-yl)-5,8-dihydropyrazolo[4',3':5,6]pyrano[2,3d]-[1,2,3]triazine (9) was obtained from 8 with pyrrole as pale yellow powder, 5-(4-fluorophenyl-6-methyl-4-morpholin-4-yl)-5,8-dihydropyrazolo-[4',3':5,6]pyrano-[2,3-d][1,2,3]triazine (10a) was obtained from compound 8 with morpholine as yellow powder, 5-(4-fluorophenyl)6-methyl-4-piperazin-1-yl)-5,8-dihydropyrazolo[4',3':5,6]pyrano[2,3-d]-[1,2,3]triazine (10b) was obtained from 8 with piperazine as brown powder, and 5-(4-fluorophenyl)-6-methyl-4-methylpiperazin-1-yl)-5,8-dihydropyrazolo[4',3':5,6]pyrano [2,3-d][1,2,3]triazine (10c) was obtained from 8 with $N$-methylpiperazine as gray powder.

4-(4-Fluorophenyl)-N-[(1Z)-(4-substituted phenyl)methylene]-5-imino-3-methyl-1,4-dihydro-pyrazolo[4',3':5,6]pyrano[2,3-d]pyrimidin-6(5H)-amine $(\mathbf{1 1} \boldsymbol{a}, \boldsymbol{b})$. General procedure. - A mixture of $2(0.31 \mathrm{~g}, 1.0 \mathrm{mmol})$, with appropriate aldehyde ( $p$-fluorobenzaldehyde or 4-methoxy benzaldehyde) $(10 \mathrm{mmol})$, piperidine $(0.5 \mathrm{~mL})$ and dioxane $(30 \mathrm{~mL})$ was refluxed for 6 hours. The precipitate was filtered off and washed several times with cold EtOH. The solid was recrystallized from the appropriate solvent.

4-(4-Fluorophenyl)-N-[(1Z)-(4-fluorophenyl)methylene]-5-imino-3-methyl-1,4-dihydro-pyrazolo[4',3':5,6]pyrano[2,3- $d]$ pyrimidin-6(5H)-amine (11a) was obtained from 2 with $p$-flurobenzaldehyde as pale yellow powder, and 4-(4-fluorophenyl)-5-imino- $N$-[(1Z)-(4methoxyphenyl)methylene]-3-methyl-1,4-dihydro-pyrazolo[4',3':5,6]pyrano[2,3-d]pyrimidin-6(5H)-amine (11b) was obtained from 2 with 4-methoxy-benzaldehyde as pale brown powder

11-(4-Fluorophenyl)-2-(4-substituted phenyl)-10-methyl-8,11-dihydropyrazolo[4',3':5,6]pyrano-[3,2-e][1,2,4]triazolo[1,5-c]pyrimidine $(\mathbf{1 2} \boldsymbol{a}, \boldsymbol{b})$. General procedure. - Compounds $\mathbf{1 1} \mathbf{a}, \mathbf{b}$ where to come with alumina-supported calcium hypochlorite $\mathrm{Ca}(\mathrm{OCl})_{2} / \mathrm{Al}_{2} \mathrm{O}_{3}=1: 1$, ground mixture, stirred overnight and the reaction mixture was quenched in water $(50 \mathrm{~mL})$.

2,11-Bis(4-fluorophenyl)-10-methyl-8,11-dihydropyrazolo[4',3':5,6]pyrano[3,2-e][1,2,4]-triazolo[1,5-c]pyrimidine (12a) was obtained from 11a as pale gray powder, while 11-(4-fluorophenyl)-2-(4-methoxyphenyl)-10-methyl-8,11-dihydropyrazolo[4',3':5,6]pyrano$[3,2-e][1,2,4]$ triazolo[1,5-c]pyrimidine $\mathbf{( 1 2} \mathbf{b})$ was obtained from $\mathbf{1 1 b}$ as pale yellow powder.

11-(4-Fluorophenyl)-10-methyl-8,11-dihydropyrazolo[4',3':5,6]pyrano[3,2-e][1,2,4]triazolo[1,5-c]pyrimidine-2(3H)-thione (13). - To a solution of $2(0.31 \mathrm{~g}, 10 \mathrm{mmol}$ in dry pyridine (30 $\mathrm{mL}), \mathrm{CS}_{2}(20 \mathrm{mmol})$ was added and the reaction mixture was heated under reflux for $8 \mathrm{~h}$. After cooling, the reaction mixture was poured onto ice/ $\mathrm{HCl}$ mixture and the solid that separated was washed with cold water and filtered off to give $\mathbf{1 3}$ as yellow powder.

2-[4-(4-Fluorophenyl)-5-imino-3-methyl-1,4-dihydropyrazolo[4',3':5,6]pyrano[2,3-d]pyrimidin- $6(5 \mathrm{H})-y l]-1 H$-isoindole-1,3(2H)-dione (14). - To a solution of $2(0.31 \mathrm{~g}, 10 \mathrm{mmol})$ in $n$ - $\mathrm{BuOH}$, $10 \mathrm{mmol}$ of phathalic acid anhydride was added. The reaction mixture was left overnight and the solid that formed (14) was collected as pale brown powder.

(2Z)-2-\{[4-(4-fluorophenyl)-5-imino-3-methyl-1,4-dihydropyrazolo[4',3':5,6]-pyrano[2,3-d] pyrimidin-6(5H)-yllimino\}-1,2-dihydro-3H-indol-3-one (15). - A solution of 2 (0.31 g, $10 \mathrm{mmol})$ in dioxane $(10 \mathrm{~mL})$ was stirred with isatin $(15 \mathrm{mmol})$ for 24 hours at room temperature. The product that separated out (15) as orange powder was filtered off. 
H. N. Hafez et al.: Facile heterocyclic synthesis and antimicrobial activity of polysubstituted and condensed pyrazolopyranopyrimidine and pyrazolopyranotriazine derivatives, Acta Pharm. 65 (2015) 399-412.

\section{Pharmacological screening}

Antimicrobial activity. - Antimicrobial activities of the newly synthesized compounds were tested in vitro for activity against the following bacteria: Gram-positive bacteria Streptococcus lactis NCTC-1030, Staphylococcus aureus NCTC-4493, Enterococcus faecalis NCTC4737, Gram-negative bacteria Escherichia coli ATCC-1416, Pseudomonas aeruginosa ATCC2642, Klebsiella pneumoniae ATCC-1081, and fungal strains Candida albicans ATCC- 14154, Aspergillus flavus ATCC-23554 and Ganoderma lucidum ATCC-33455. All microorganisms were purchased from the American Type Culture Collection (Manassas, USA). The newly synthesized compounds (2-15) were dissolved in DMSO and tested for antimicrobial activity with the agar disk diffusion technique (21), using a 1-cm microplate-well diameter and a solution of $100 \mu \mathrm{g} \mathrm{mL} \mathrm{m}^{-1}$ of the test compound. Compound-impregnated disks were placed on an agar plate containing a standard suspension of microorganisms. The plate was incubated for $24 \mathrm{~h}$ at $37^{\circ} \mathrm{C}$. Diameters of the zones of inhibition were measured with calipers or automated scanners and were compared with those of the standards. Norfloxacin $\left(0.16 \mu \mathrm{mol} \mathrm{mL} \mathrm{m}^{-1}\right)$ and fluconazole $\left(6.5 \times 10^{-3} \mu \mathrm{mol} \mathrm{mL} \mathrm{mL}^{-1}\right)$ were used as reference drugs for antibacterial and antifungal activity, respectively.

For determination of minimum inhibitory concentrations (MIC) (22) by the serial plate dilution method, $5 \mathrm{mg}$ of each test compound was dissolved in $1 \mathrm{~mL}$ of dimethylsulfoxide (DMSO) to prepare the stock solution. Serial dilutions were prepared from the stock solution. The plates were incubated at $37^{\circ} \mathrm{C}$ for $24 \mathrm{~h}$. MIC was the lowest concentration $(\mu \mathrm{mol}$ $\mathrm{mL}^{-1}$ ) of the test compound that resulted in no visible growth on the plates. DMSO was used as a solvent control to ensure that the solvent had no effect on bacterial growth. The results of antimicrobial activities are summarized in Tables I and II.

\section{RESULTS AND DISCUSSION}

\section{Chemistry}

The bifunctional compound $\mathbf{1}$ was used for the synthesis of pyrazolopyranopyrimidine derivatives by the reaction with different reagents. Thus, the treatment of 6-amino-3methyl-4-(substituted-phenyl)-1,4-dihydropyrano[2,3-c]pyrazole-5-carbonitrile (1), which is the starting material (23) for the synthesis of polycyclic fused ring systems, with triethylorthoformate followed by hydrazine hydrate afforded three fused rings, namely, 4-(4-fluorophenyl)-5-imino-3-methyl-1,4-dihydropyrazolo[4',3':5,6]pyrano[2,3-d]pyrimidin-6(5H)-amine (2). Also, the reaction of 1 with formic acid and/or acetic acid afforded pyranopyrimidine derivatives $\mathbf{3} \mathbf{a}, \mathbf{b}$. In addition, the interaction of $\mathbf{1}$ with phenylisocyanate consumed two moles of the reagent to furnish $\mathrm{N}$-[(5Z)-4-(4-fluorophenyl)-3-methyl-7-oxo-6-phenyl-4,6,7,8-tetrahydropyrazolo[4,'3':5,6]-pyrano[2,3-d]pyrimidine-5(1H)-ylidene]benzamide (4). Heating of 1 with ammonium thiocyanate in boiling acetic acid gave pyranopyrimidinethione derivatives (5). The treatment of $\mathbf{1}$ with formamide in the presence of formic acid and dimethylformamide gave compound $\mathbf{6}$. The structure of $\mathbf{6}$ was confirmed via inspection of elemental analysis and spectral data. Its ${ }^{1} \mathrm{H}$ NMR spectrum revealed $\delta$ at 5.00 (s, pyran- $H$ ), 7.19 (d, 2H, Ar- $H$ ), 7.80 (d, 2H, Ar- $H$ ), 8.10 (s, CH pyrimidine) and 10.29, 11.60 ppm (2 NH). Compound 4-(4-fluorophenyl)-3-methyl-1,4,6,7,8,9-hexahydropyrazolo-[4',3':5,6]pyrano[2,3-b]quinolin-5-amine (7) was obtained in good yield (see 


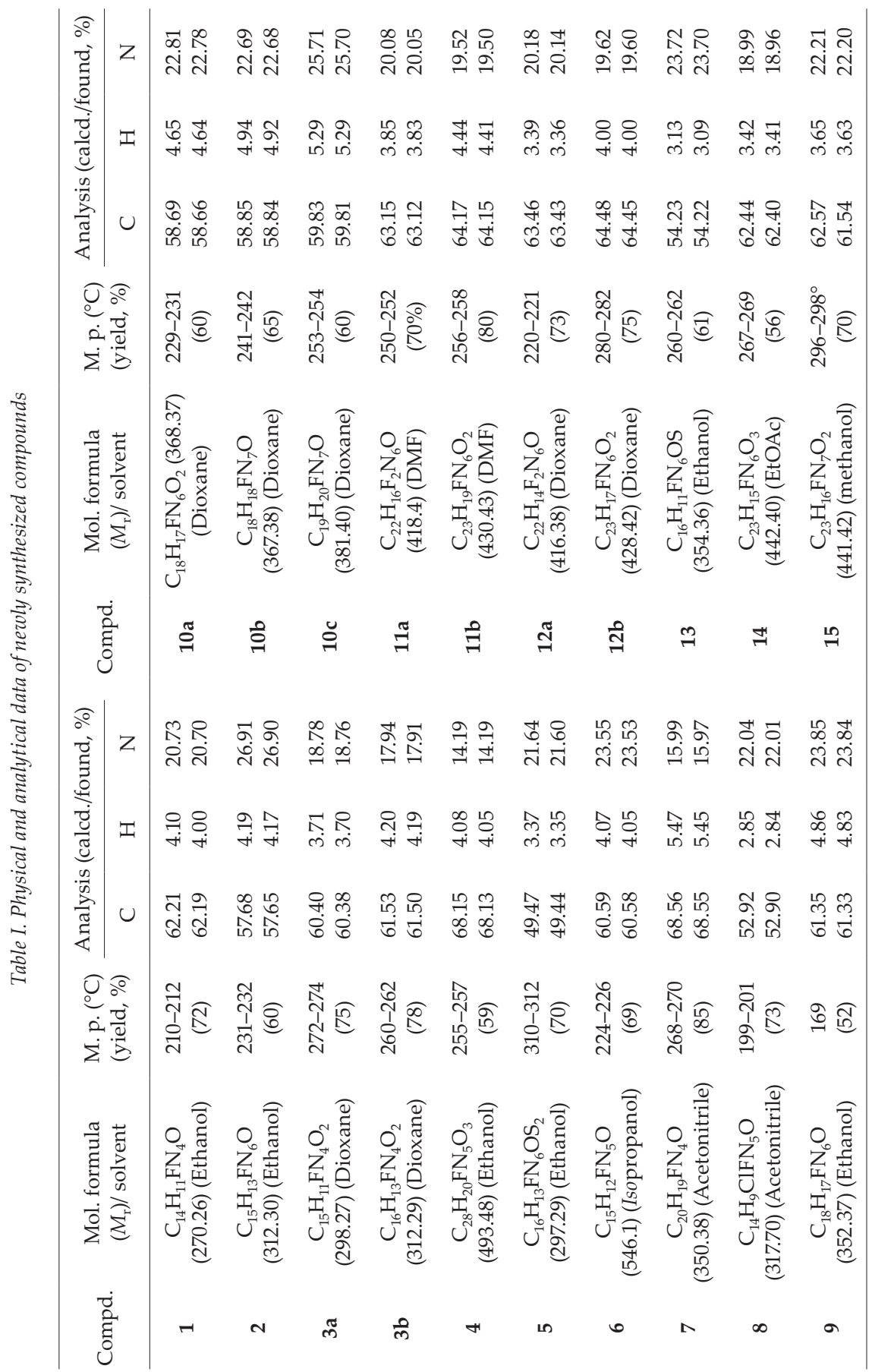


H. N. Hafez et al.: Facile heterocyclic synthesis and antimicrobial activity of polysubstituted and condensed pyrazolopyranopyrimidine and pyrazolopyranotriazine derivatives, Acta Pharm. 65 (2015) 399-412.

Table II. Spectral data of newly synthesized compounds

\begin{tabular}{|c|c|c|c|}
\hline Compd. & $\begin{array}{c}\mathrm{IR}(\mathrm{KBr}) \\
\left(v_{\max }, \mathrm{cm}^{-1}\right)\end{array}$ & $\begin{array}{l}\mathrm{MS} \\
(m / z)\end{array}$ & $\begin{array}{l}{ }^{1} \mathrm{H},{ }^{13} \mathrm{C} \text { NMR }\left(\mathrm{DMSO}-d_{6}\right) \\
(\delta, \mathrm{ppm})\end{array}$ \\
\hline 1 & $\begin{array}{l}3370,3173 \\
3050,2922 \\
2202,1640\end{array}$ & $\begin{array}{l}\text { MS } 270 \\
\left(\mathrm{M}^{+}, 72 \%\right)\end{array}$ & $\begin{array}{l}2.20\left(\mathrm{~s}, 3 \mathrm{H}, \mathrm{CH}_{3}\right), 4.94(\mathrm{~s}, \text { pyran- } H), 7.23(\mathrm{~d}, 2 \mathrm{H}, \mathrm{Ar}-H), 7.90(\mathrm{~d}, 2 \mathrm{H}, \\
\text { Ar- } H), 10.24,10.52\left(2 \mathrm{brs}, \mathrm{D}_{2} \mathrm{O} \text { exchangeable-NH) }\right.\end{array}$ \\
\hline 2 & 3370,1615 & $\begin{array}{l}\text { MS } 312 \\
\left(\mathrm{M}^{+}, 86 \%\right)\end{array}$ & $\begin{array}{l}2.17\left(\mathrm{~s}, 3 \mathrm{H}, \mathrm{CH}_{3}\right), 5.05(\mathrm{~s}, \text { pyran- } H), 7.23(\mathrm{~d}, 2 \mathrm{H}, \mathrm{Ar}-H), 7.72(\mathrm{~d}, 2 \mathrm{H}, \\
\text { Ar- } H), 8.01(\mathrm{~s}, 1 \mathrm{H}, \text { pyrimidine- } \mathrm{CH}) .10 .05,10.26\left(2 \mathrm{brs}, \mathrm{D}_{2} \mathrm{O}\right. \\
\text { exchangeable-NH) }\end{array}$ \\
\hline $3 a$ & $\begin{array}{l}3300,1690, \\
1620\end{array}$ & $\begin{array}{l}\text { MS } 298 \\
\left(\mathrm{M}^{+}, 83 \%\right)\end{array}$ & $\begin{array}{l}2.23\left(\mathrm{~s}, 3 \mathrm{H}, \mathrm{CH}_{3}\right), 5.01(\mathrm{~s}, \text { pyran- } H), 8.58(1 \mathrm{H}, \mathrm{s}, \text { pyrimidine- } \mathrm{CH}) \\
7.23(\mathrm{~d}, 2 \mathrm{H}, \mathrm{Ar}-H), 7.78(\mathrm{~d}, 2 \mathrm{H}, \mathrm{Ar}-\mathrm{H}), 8.09(\mathrm{~s}, 1 \mathrm{H}, \text { pyrimidine- } \mathrm{CH}) \text {. } \\
10.20,10.62\left(2 \mathrm{brs}, \mathrm{D}_{2} \mathrm{O} \text { exchangeable- } \mathrm{N} H\right)\end{array}$ \\
\hline $3 b$ & $\begin{array}{l}3300,1690, \\
1620\end{array}$ & $\begin{array}{l}\text { MS } 312 \\
\left(\mathrm{M}^{+}, 74 \%\right)\end{array}$ & $\begin{array}{l}2.20\left(\mathrm{~s}, 3 \mathrm{H}, \mathrm{CH}_{3}\right), 2.24\left(\mathrm{~s}, 3 \mathrm{H}, \mathrm{CH}_{3}\right) 5.05(\mathrm{~s}, \text { pyran- } H), 7.23(\mathrm{~d}, 2 \mathrm{H}, \\
\text { Ar- } H), 7.78(\mathrm{~d}, 2 \mathrm{H}, \mathrm{Ar}-H), 10.20,10.62\left(2 \mathrm{brs}, \mathrm{D}_{2} \mathrm{O} \text { exchangeable-NH) }\right.\end{array}$ \\
\hline 4 & $\begin{array}{l}3379,3247 \\
1735,1689\end{array}$ & $\begin{array}{l}\text { MS } 508 \\
\left(\mathrm{M}^{+}, 65 \%\right)\end{array}$ & $\begin{array}{l}2.20\left(\mathrm{~s}, 3 \mathrm{H}, \mathrm{CH}_{3}\right), 4.99(\mathrm{~s}, \text { pyran- } H), 6.90-7.01(\mathrm{~m}, 5 \mathrm{H}, \mathrm{Ar}-\mathrm{H}), 7.37-7.90 \\
(\mathrm{~m}, 5 \mathrm{H}, \mathrm{Ar}-\mathrm{H}), 8.09(\mathrm{~d}, 2 \mathrm{H}, \mathrm{Ar}-\mathrm{H}), 8.20(\mathrm{~d}, 2 \mathrm{H}, \mathrm{Ar}-H), 8.35(\mathrm{~s}, 1 \mathrm{H}, \\
\text { pyrimidine-CH), } 10.65,11.53,14.03,\left(3 \mathrm{~s}, 3 \mathrm{H}, \mathrm{D}_{2} \mathrm{O} \text { exchangeable } 3 \mathrm{NH}\right.\end{array}$ \\
\hline 5 & $\begin{array}{l}3424,2923 \\
1230,1235\end{array}$ & $\begin{array}{l}\text { MS } 388 \\
\left(\mathrm{M}^{+}, 85 \%\right)\end{array}$ & $\begin{array}{l}2.23\left(\mathrm{~s}, 3 \mathrm{H}, \mathrm{CH}_{3}\right), 4.95(\mathrm{~s}, \text { pyran- } H), 7.23(\mathrm{~d}, 2 \mathrm{H}, \text { Ar- } H), 7.90(\mathrm{~d}, 2 \mathrm{H}, \\
\text { Ar- } H), 10.24,10.52,11.43,12.03,\left(4 \mathrm{brs}, \mathrm{D}_{2} \mathrm{O} \text { exchangeable- } \mathrm{N} H\right)\end{array}$ \\
\hline 6 & 3370,1622 & $\begin{array}{l}\text { MS } 297 \\
\left(\mathrm{M}^{+}, 80 \%\right)\end{array}$ & $\begin{array}{l}2.19\left(\mathrm{~s}, 3 \mathrm{H}, \mathrm{CH}_{3}\right), 5.00(\mathrm{~s}, \text { pyran- } H), 7.19(\mathrm{~d}, 2 \mathrm{H}, \mathrm{Ar}-H), 7.80(\mathrm{~d}, 2 \mathrm{H}, \\
\text { Ar- } H), 8.10(\mathrm{~s}, 1 \mathrm{H}, \text { pyrimidine- } \mathrm{CH}), 10.29,11.60\left(2 \mathrm{brs}, \mathrm{D}_{2} \mathrm{O}\right. \\
\text { exchangeable-NH) }\end{array}$ \\
\hline 7 & 3320,3205 & $\begin{array}{l}\text { MS } 350 \\
\left(\mathrm{M}^{+}, 75 \%\right)\end{array}$ & $\begin{array}{l}1.76\left(\mathrm{~m}, 4 \mathrm{H}, 2 \mathrm{CH}_{2}\right), 2.20\left(\mathrm{~s}, 3 \mathrm{H}, \mathrm{CH}_{3}\right), 2.45\left(\mathrm{~m}, 2 \mathrm{H}, \mathrm{CH}_{2}\right), 2.70(\mathrm{~m}, \\
\left.2 \mathrm{H}, \mathrm{CH}_{2}\right), 4.98(\mathrm{~s}, \text { pyran- } \mathrm{H}), 6.22\left(\mathrm{~s}, 2 \mathrm{H}, \mathrm{NH}_{2}\right), 7.20(\mathrm{~d}, 2 \mathrm{H}, \mathrm{Ar}-\mathrm{H}), \\
7.80(\mathrm{~d}, 2 \mathrm{H}, \text { Ar- } H), 10.22,\left(\mathrm{br}, 1 \mathrm{NH}, \mathrm{D}_{2} \mathrm{O} \text { exchangeable }\right)\end{array}$ \\
\hline 8 & 3310,1622 & $\begin{array}{l}\text { MS } 317 \\
\left(\mathrm{M}^{+}, 70 \%\right)\end{array}$ & $\begin{array}{l}2.21\left(\mathrm{~s}, 3 \mathrm{H}, \mathrm{CH}_{3}\right), 5.12(\mathrm{~s}, \text { pyran- } H), 7.15(\mathrm{~d}, 2 \mathrm{H}, \mathrm{Ar}-H), 7.60(\mathrm{~d}, 2 \mathrm{H}, \\
\text { Ar- } H), 10.35\left(\mathrm{br}, 1 \mathrm{NH}, \mathrm{D}_{2} \mathrm{O} \text { exchangeable }\right)\end{array}$ \\
\hline 9 & 3315 & $\begin{array}{l}\text { MS } 352 \\
\left(\mathrm{M}^{+}, 53 \%\right)\end{array}$ & $\begin{array}{l}2.19\left(\mathrm{~s}, 3 \mathrm{H}, \mathrm{CH}_{3}\right), 2.58\left(\mathrm{~m}, 4 \mathrm{H}, 2 \mathrm{CH}_{2}\right), 3.56\left(\mathrm{~m}, 2 \mathrm{H}, \mathrm{CH}_{2}\right), 3.80(\mathrm{~m}, \\
\left.2 \mathrm{H}, \mathrm{CH}_{2}\right), 4.98(\mathrm{~s}, \text { pyran- } H), 7.21(\mathrm{~d}, 2 \mathrm{H}, \mathrm{Ar}-\mathrm{H}), 7.80(\mathrm{~d}, 2 \mathrm{H}, \mathrm{Ar}-\mathrm{H}), \\
10.34,\left(\mathrm{br}, 1 \mathrm{NH}, \mathrm{D}_{2} \mathrm{O} \text { exchangeable }\right)\end{array}$ \\
\hline $10 a$ & 3310 & $\begin{array}{l}\text { MS } 368 \\
\left(\mathrm{M}^{+}, 65 \%\right)\end{array}$ & $\begin{array}{l}2.15\left(\mathrm{~s}, 3 \mathrm{H}, \mathrm{CH}_{3}\right), 3.47\left(\mathrm{~m}, 4 \mathrm{H}, 2 \mathrm{CH}_{2}\right), 3.76\left(\mathrm{~m}, 4 \mathrm{H}, 2 \mathrm{CH}_{2}\right), 5.01 \\
(\mathrm{~s}, \text { pyran- } \mathrm{H}), 7.15(\mathrm{~d}, 2 \mathrm{H}, \mathrm{Ar}-\mathrm{H}), 7.74(\mathrm{~d}, 2 \mathrm{H}, \mathrm{Ar}-\mathrm{H}), 10.40(\mathrm{br}, 1 \mathrm{NH}, \\
\mathrm{D}_{2} \mathrm{O} \text { exchangeable) }\end{array}$ \\
\hline $10 b$ & 3315 & $\begin{array}{l}\text { MS } 367 \\
\left(\mathrm{M}^{+}, 70 \%\right)\end{array}$ & $\begin{array}{l}2.17\left(\mathrm{~s}, 3 \mathrm{H}, \mathrm{CH}_{3}\right), 2.48\left(\mathrm{brs}, 4 \mathrm{H} \text {, piperazinyl } 2 \mathrm{NCH}_{2}\right), 3.29(\mathrm{brs}, 4 \mathrm{H}, \\
\left.\text { piperazinyl } 2 \mathrm{NCH}_{2}\right), 5.05(\mathrm{~s}, \text { pyran- } \mathrm{H}), 7.08(\mathrm{~d}, 2 \mathrm{H}, \mathrm{Ar}-\mathrm{H}), 7.68(\mathrm{~d}, \\
2 \mathrm{H}, \mathrm{Ar}-\mathrm{H}), 10.25,\left(\mathrm{br}, 1 \mathrm{NH}, \mathrm{D}_{2} \mathrm{O} \text { exchangeable) }\right.\end{array}$ \\
\hline $10 c$ & 3312 & $\begin{array}{l}\text { MS } 381 \\
\left(\mathrm{M}^{+}, 67 \%\right)\end{array}$ & $\begin{array}{l}2.13\left(\mathrm{~s}, 3 \mathrm{H}, \mathrm{CH}_{3}\right), 2.33\left(\mathrm{~s}, 3 \mathrm{H} \text {, piperazinyl } \mathrm{NCH}_{3}\right), 2.53(\mathrm{brs}, 4 \mathrm{H}, \\
\left.\text { piperazinyl } 2 \mathrm{NCH}_{2}\right), 3.36\left(\mathrm{brs}, 4 \mathrm{H} \text {, piperazinyl } 2 \mathrm{NCH}_{2}\right), 5.00(\mathrm{~s}, \\
\text { pyran- } \mathrm{H}), 7.01(\mathrm{~d}, 2 \mathrm{H}, \mathrm{Ar}-\mathrm{H}), 7.80(\mathrm{~d}, 2 \mathrm{H}, \mathrm{Ar}-\mathrm{H}), 10.09,(\mathrm{br}, 1 \mathrm{NH}, \\
\mathrm{D}_{2} \mathrm{O} \text { exchangeable) }\end{array}$ \\
\hline $11 a$ & 3325,1625 & $\begin{array}{l}\text { MS } 418 \\
\left(\mathrm{M}^{+}, 90 \%\right)\end{array}$ & $\begin{array}{l}2.30\left(\mathrm{~s}, 3 \mathrm{H}, \mathrm{CH}_{3}\right), 5.10(\mathrm{~s}, \text { pyran- } \mathrm{H}), 6.80\left(\mathrm{~s}, 2 \mathrm{H}, \mathrm{NH}_{2}\right), 7.25(\mathrm{~d}, 2 \mathrm{H}, \\
\mathrm{Ar}-\mathrm{H}), 7.80(\mathrm{~d}, 2 \mathrm{H}, \mathrm{Ar}-\mathrm{H}), 7.57(\mathrm{~d}, 2 \mathrm{H}, \mathrm{Ar}-\mathrm{H}), 7.94(\mathrm{~d}, 2 \mathrm{H}, \mathrm{Ar}-\mathrm{H}), \\
8.39(\mathrm{~s}, 1 \mathrm{H}, \text { pyrimidine- } \mathrm{CH}), 8.90(\mathrm{~s}, 1 \mathrm{H}, \mathrm{N}=\mathrm{CH}), 10.09(\mathrm{br}, 1 \mathrm{NH}, \\
\mathrm{D}_{2} \mathrm{O} \text { exchangeable) }\end{array}$ \\
\hline
\end{tabular}


Table II. continued

\begin{tabular}{|c|c|c|c|}
\hline Compd. & $\begin{array}{c}\mathrm{IR}(\mathrm{KBr}) \\
\left(v_{\max }, \mathrm{cm}^{-1}\right)\end{array}$ & $\begin{array}{l}\text { MS } \\
(m / z)\end{array}$ & $\begin{array}{c}\left.{ }^{1} \mathrm{H},{ }^{13} \mathrm{C} \text { NMR (DMSO- } d_{6}\right) \\
(\delta, \mathrm{ppm})\end{array}$ \\
\hline $11 b$ & 3315,1615 & $\begin{array}{l}\text { MS) } 430 \\
\left(\mathrm{M}^{+}, 85 \%\right)\end{array}$ & $\begin{array}{l}2.35\left(\mathrm{~s}, 3 \mathrm{H}, \mathrm{CH}_{3}\right), 3.59\left(\mathrm{~s}, 3 \mathrm{H}, \mathrm{OCH}_{3}\right), 5.06(\mathrm{~s}, \text { pyran- } H), 6.82(\mathrm{~s}, 1 \mathrm{H}, \\
\mathrm{NH}), 7.30(\mathrm{~d}, 2 \mathrm{H}, \mathrm{Ar}-\mathrm{H}), 7.78(\mathrm{~d}, 2 \mathrm{H}, \mathrm{Ar}-\mathrm{H}), 7.60(\mathrm{~d}, 2 \mathrm{H}, \mathrm{Ar}-H), 7.99 \\
(\mathrm{~d}, 2 \mathrm{H}, \mathrm{Ar}-\mathrm{H}), 8.35(\mathrm{~s}, 1 \mathrm{H}, \text { pyrimidine- } \mathrm{CH}), 9.25(\mathrm{~s}, 1 \mathrm{H}, \mathrm{N}=\mathrm{CH}), \\
10.05\left(\mathrm{br}, 1 \mathrm{NH}, \mathrm{D}_{2} \mathrm{O} \text { exchangeable) }\right.\end{array}$ \\
\hline $12 a$ & 3320,1630 & $\begin{array}{l}\text { (MS) } 416 \\
\left(\mathrm{M}^{+}, 90 \%\right)\end{array}$ & $\begin{array}{l}2.22\left(\mathrm{~s}, 3 \mathrm{H}, \mathrm{CH}_{3}\right), 5.10(\mathrm{~s}, \text { pyran- } H), 7.20(\mathrm{~d}, 2 \mathrm{H}, \mathrm{Ar}-H), 7.85(\mathrm{~d}, 2 \mathrm{H}, \\
\text { Ar- } H), 7.48(\mathrm{~d}, 2 \mathrm{H}, \mathrm{Ar}-H), 8.00(\mathrm{~d}, 2 \mathrm{H}, \mathrm{Ar}-\mathrm{H}), 8.30(\mathrm{~s}, 1 \mathrm{H}, \text { pyrimi- } \\
\text { dine-CH) } 10.20\left(\mathrm{br}, 1 \mathrm{NH}, \mathrm{D}_{2} \mathrm{O} \text { exchangeable }\right)\end{array}$ \\
\hline $12 b$ & 3318,1625 & $\begin{array}{l}\text { (MS) } 428 \\
\left(\mathrm{M}^{+}, 90 \%\right)\end{array}$ & $\begin{array}{l}2.20\left(\mathrm{~s}, 3 \mathrm{H}, \mathrm{CH}_{3}\right), 3.60\left(\mathrm{~s}, 3 \mathrm{H}, \mathrm{OCH}_{3}\right), 5.05(\mathrm{~s}, \text { pyran- } H), 7.22(\mathrm{~d}, 2 \mathrm{H}, \\
\text { Ar- }-\mathrm{H}), 7.87(\mathrm{~d}, 2 \mathrm{H}, \mathrm{Ar}-H), 7.44(\mathrm{~d}, 2 \mathrm{H}, \mathrm{Ar}-\mathrm{H}), 8.05(\mathrm{~d}, 2 \mathrm{H}, \mathrm{Ar}-\mathrm{H}), \\
8.35\left(\mathrm{~s}, 1 \mathrm{H}, \text { pyrimidine-CH), } 10.23\left(\mathrm{br}, 1 \mathrm{NH}, \mathrm{D}_{2} \mathrm{O} \text { exchangeable }\right)\right.\end{array}$ \\
\hline 13 & 1453,3400 & $\begin{array}{l}\text { (MS) } 353 \\
\left(\mathrm{M}^{+}, 60 \%\right)\end{array}$ & $\begin{array}{l}2.19\left(\mathrm{~s}, 3 \mathrm{H}, \mathrm{CH}_{3}\right), 5.05(\mathrm{~s}, \text { pyran- } H), 7.30(\mathrm{~d}, 2 \mathrm{H}, \mathrm{Ar}-\mathrm{H}), 7.80(\mathrm{~d}, 2 \mathrm{H}, \\
\text { Ar- } H), 8.15(\mathrm{~s}, 1 \mathrm{H}, \text { pyrimidine- } \mathrm{CH}), 10.90,11.52\left(2 \mathrm{brs}, 2 \mathrm{H}, \mathrm{D}_{2} \mathrm{O}\right. \\
\text { exchangeable-2NH) }\end{array}$ \\
\hline 14 & $\begin{array}{l}1685,1737, \\
3118\end{array}$ & $\begin{array}{l}\text { (MS) } 442 \\
\left(\mathrm{M}^{+}, 60 \%\right)\end{array}$ & $\begin{array}{l}2.30\left(\mathrm{~s}, 3 \mathrm{H}, \mathrm{CH}_{3}\right), 4.98(\mathrm{~s}, \text { pyran- } H), 7.14-7.20(\mathrm{~m}, 2 \mathrm{H}, \mathrm{Ar}-\mathrm{H} \text { and } \\
\text { pyrimidine- } H), 7.54-7.81(\mathrm{~m}, 3 \mathrm{H}, \mathrm{Ar}-H), 7.99(\mathrm{~d}, 2 \mathrm{H}, \mathrm{Ar}-H), 8.10 \\
(\mathrm{~d}, 2 \mathrm{H}, \mathrm{Ar}-H), 9.05,10.11\left(2 \mathrm{brs}, 2 \mathrm{H}, \mathrm{D}_{2} \mathrm{O} \text { exchangeable-NH) }\right.\end{array}$ \\
\hline 15 & $\begin{array}{l}3479,1700 \\
1619\end{array}$ & $\begin{array}{l}\text { (MS) } 441 \\
\left(\mathrm{M}^{+}, 75 \%\right)\end{array}$ & $\begin{array}{l}2.20\left(\mathrm{~s}, 3 \mathrm{H}, \mathrm{CH}_{3}\right), 4.95(\mathrm{~s}, \text { pyran- } H), 7.37-7.86(\mathrm{~m}, 4 \mathrm{H}, \mathrm{Ar}-\mathrm{H}), 8.07 \\
(\mathrm{~d}, 2 \mathrm{H}, \mathrm{Ar}-\mathrm{H}), 8.16(\mathrm{~d}, 2 \mathrm{H}, \text { Ar- } \mathrm{H}), 8.30(\mathrm{~s}, 1 \mathrm{H}, \text { pyrimidine- } \mathrm{CH}) \\
10.65,14.20\left(2 \mathrm{~s}, 2 \mathrm{H}, \mathrm{D}_{2} \mathrm{O} \text { exchangeable } 2 \mathrm{NH}\right)\end{array}$ \\
\hline
\end{tabular}

Scheme 1) by the Friedlander reaction on pyranopyrazole 1 (24) by heating compound 1 with cyclohexanone in dichloroethane, aluminium chloride and THF as solvent and reflux of reaction mixture for $15 \mathrm{~h}$.

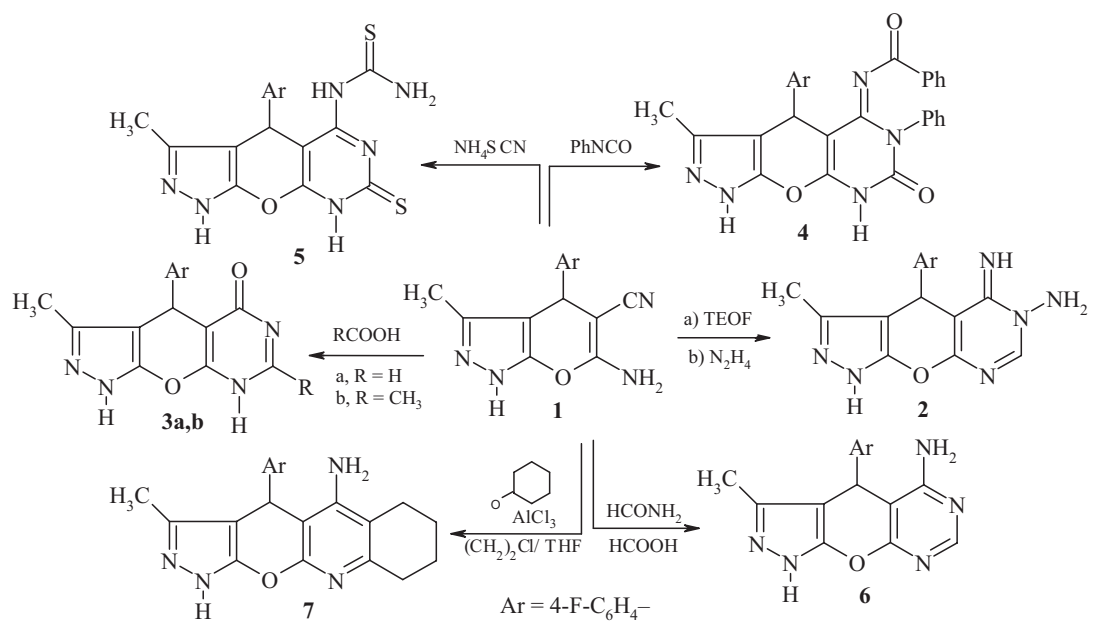

Scheme 1 
Amino-cyanopyranopyrazole 1 could also be used to synthesize pyrazolopyranotriazine 8. The 4- chlorotriazine ring was prepared via a one-pot process of diazotation and dediazo-chlorination. The reactivity of chlorine atom in 4-chloro-5-(4-fluorophenyl)-6methyl-5,8-dihydropyrazolo-[4',3':5,6]pyrano[2,3- $d][1,2,3]$ triazine (8) appeared when compound 8 was subject to the action of secondary amine, namely, pyrole, morpholine, piperazine and $\mathrm{N}$-methylpiperazine. The chlorine atom was displaced to form the corresponding compounds $\mathbf{9}$ and $\mathbf{1 0 a}-\mathbf{c}$, respectively (Scheme 2).

According to the literature $(25,26)$, compounds having azomethine linkage exhibit $E / Z$ geometrical isomerism around $\mathrm{C}=\mathrm{N}$ double bond and can exist as cis/trans amide conformers (26). Moreover, hydrazones were proven to exist in higher percentage in DMSO- $d_{6}$ solution in the form of geometrical $E$ isomers. Thus, compound 2 on treatment with the appropriate aromatic aldehyde, namely $p$-fluorobenzaldehyde/anisaldehyde, by refluxing with ethanol in the presence of a catalytic amount of piperidine gave 4-(4-fluorophenyl)$N$-[(1E)-(4-substitutedphenyl)-methyl-ene]-5-imino-3-methyl-1,4-dihydropyrazolo[4', $\left.3^{\prime}: 5,6\right]$ pyrano[2,3-d]pyrimidin-6(5H)-amines $(\mathbf{1 1} \mathbf{a}, \mathbf{b})$. Also, NMR spectra of compounds 11 recorded in DMSO- $d_{6}$ solution revealed that all compounds existed as $E$ geometrical isomers. Oxidative cyclization of the resultant hydrazone derivatives $\mathbf{1 1} \mathbf{a}, \mathbf{b}$ to $\mathbf{1 2} \mathbf{a}, \mathbf{b}$ was achieved by using alumina-supported calcium hypochlorite $\left(\mathrm{Ca}(\mathrm{OCl})_{2} / \mathrm{Al}_{2} \mathrm{O}_{3}=1: 1\right.$, ground mixture) as a new oxidant.

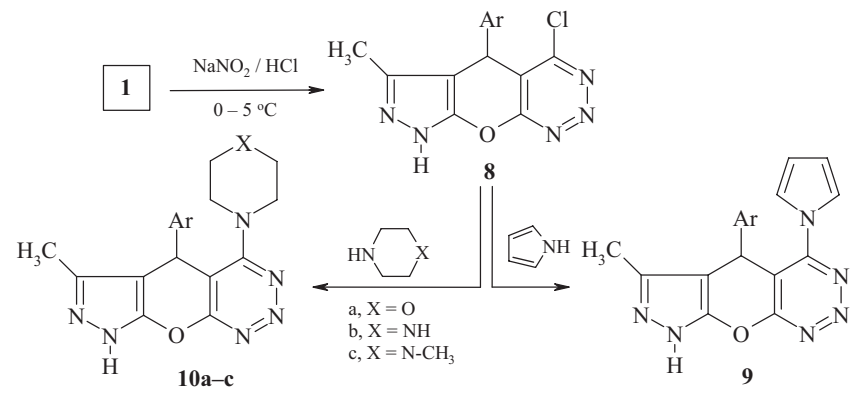

Scheme 2

For instance, a maximum yield of $73 \%$ for $\mathbf{1 2 a}$ and $75 \%$ for $\mathbf{1 2 b}$ in $2 \mathrm{~h}$ was achieved with the 1:3 molar ratio of hydrazone to calcium hypochlorite. The use of alumina-supported calcium hypochlorite as a heterogeneous oxidant in this reaction has the advantage of an enhanced reaction rate and yield (see Scheme 3).

Furthermore, the hydrazide derivative 2 reacted with carbon disulfide and $\mathrm{NaOH}$ in ethanol to afford 11-(4-fluorophenyl)-10-methyl-8,11-dihydropyrazolo[4,'3':5,6]pyrano[3,2-e] [1,2,4]tria-zolo[1,5-c]pyrimidine-2(3H)-thione (13) (Scheme 3).

In the present work, we wish to point out the reaction of hydrazino derivative 2 with phthalic anhydride. When compound 2 with phthalic anhydride in an oil bath, it yielded 2-phthalimidoamino derivative 14 . The reaction took place via acylation of hydrazino moiety by phthalic anhydride, followed by ring closure to give the desired product. On the other hand, stirring of compound $\mathbf{2}$ with isatin at room temperature yielded compound 15 


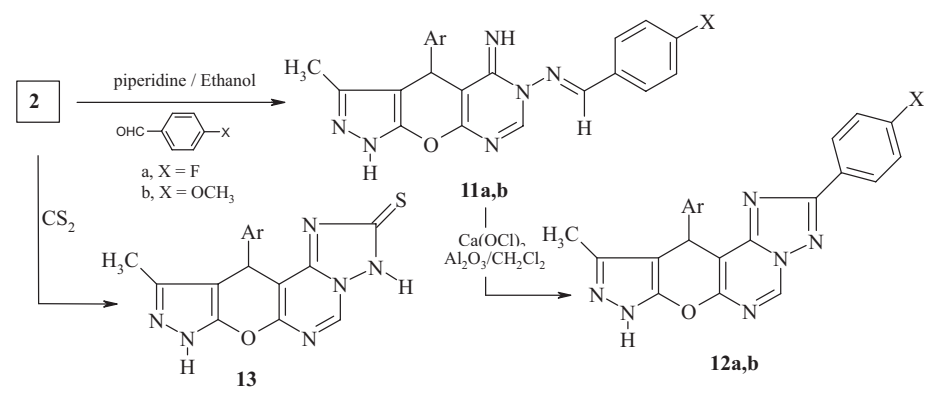

Scheme 3

(Scheme 4). The structure of the resultant compound was fully established by spectral data, which included IR, ${ }^{1} \mathrm{H}$ NMR and MS spectra. The ${ }^{1} \mathrm{H}$ NMR spectra of compound 15 revealed signals at $\delta: 2.20\left(\mathrm{~s}, 3 \mathrm{H}, \mathrm{CH}_{3}\right), 4.95(\mathrm{~s}$, pyran- $H), 7.37-7.86(\mathrm{~m}, 4 \mathrm{H}, \mathrm{Ar}-\mathrm{H}), 8.07(\mathrm{~d}, 2 \mathrm{H}$, Ar-H), $8.16(\mathrm{~d}, 2 \mathrm{H}, \mathrm{Ar}-\mathrm{H}), 8.30(\mathrm{~s}, 1 \mathrm{H}$, pyrimidine $\mathrm{CH}), 10.65$ and $14.20 \mathrm{ppm}\left(2 \mathrm{~s}, 2 \mathrm{H}, \mathrm{D}_{2} \mathrm{O}\right.$ exchangeable $2 \mathrm{NH})$.

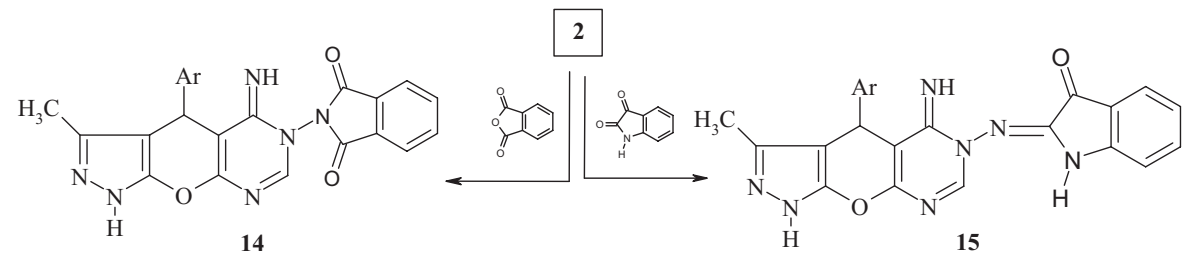

Scheme 4

\section{Antimicrobial screening and $S A R$}

The minimum inhibitory concentration values (MICs) against different Gram-positive, Gram-negative bacteria and fungi were tested. The investigations showed significant inhibitory effects against bacteria with the majority of the compounds with MIC values of 1-21 $\mu \mathrm{mol} \mathrm{mL} \mathrm{m}^{-1}$ (Table III). The antibacterial data indicated that compounds $\mathbf{2}, \mathbf{3 a}, \mathbf{b}, \mathbf{4}, \mathbf{5}, \mathbf{6}$, 11a,b, 13 and $\mathbf{1 4}$ displayed good activity against all tested bacteria. This activity can be attributed to the presence of pyrimidine moiety. Compound 2 comprised iminopyrimidine with amino group attached at $N-3$, compounds $3 \mathbf{a}, \mathbf{b}$ are pyrimidone derivatives, while compounds 4,5 are 6 are 4-amino pyrimidine and pyrimidine thione derivatives. Compounds 11a,b contain $N$-hydrazonyl, 13 triazolo thione and $14 \mathrm{~N}$-isoindol dione pyrimidine moieties. Evaluation of the antibacterial activity of synthesized compounds $\mathbf{7}$ and $\mathbf{8}$ revealed that compounds were effective against all tested bacteria due to the presence of tetrahydroquinoline moiety in $\mathbf{7}$ and 4-chlorotriazine in 8. Regarding the antibacterial activities, pyrazolopyranotriazine derivatives 9 and 10a-c were the most active compounds; their MIC values were $1-5 \mu \mathrm{mol} \mathrm{mL} \mathrm{m}^{-1}$ against Gram-positive and $3-8 \mu \mathrm{mol} \mathrm{mL}^{-1}$ against Gram-negative bacteria compared to the standard drug norfloxacin with respective MICs of $2-3$ and 
4-5 $\mu \mathrm{mol} \mathrm{mL} \mathrm{m}^{-1}$. The antimicrobial activity of compound 10c comprising $N$-methylpiperazine attached to the triazine moiety at position 4 exhibited the higher activity against $S$. lactis, E. faecalis, E. coli and K. pneumoniae, but was equipotent against S. aureus and P. aeuroginosa compared to norfloxacin, whereas compounds $\mathbf{9}$ and $\mathbf{1 0 a} \mathbf{a} \mathbf{b}$ showed promising activity against bacteria which were equal or nearly close to MIC of norfloxacin. The activity of the latter compounds can be attributed to the presence of pyrolyl in 9, morpholinyl in 10a and piperazinyl moiety in $\mathbf{1 0 b}$, attached to the pyranotriazine, which increased the antimicrobial activity against Gram-positive and Gram-negative bacteria.

On the other hand compound 12a,b displayed antibacterial activity with MIC equal or nearly close to MIC of norfloxacin, This is attributed to the presence of 4 -fluoro substituent in the phenyl ring attached to $[1,2,4]$ triazole in compound $\mathbf{1 2} \mathbf{a}$, while compound $\mathbf{1 2 b}$ had 4-methoxyphenyl. Among the synthesized hydrazone derivatives, compound 15 displayed antibacterial activity comparable to that of norfloxacin against $E$. faecalis, E. coli and $P$. aeuroginosa, and was equal or nearly close to MIC of norfloxacin against S. lactis, $S$. aureus and K. pneumoniae. This can be attributed to the presence of the indol-3-one attached to $N$-pyrimidinyl derivative.

Table III. Minimal inhibitory concentration (MIC, $\mu \mathrm{mol} \mathrm{mL}^{-1}$ ) of the synthesized compounds against bacteria

\begin{tabular}{ccccccc}
\hline \multirow{2}{*}{ Compd. } & \multicolumn{6}{c}{ Microorganism } \\
\cline { 2 - 7 } & S. lactis & S. aureus & E. faecalis & E. coli & P. aeuroginosa & K. pneumoniae \\
\hline $\mathbf{2}$ & 9 & 12 & 17 & 20 & 20 & 21 \\
$\mathbf{3 a}$ & 14 & 12 & 14 & 18 & 14 & 14 \\
$\mathbf{3 b}$ & 15 & 16 & 15 & 16 & 17 & 14 \\
$\mathbf{4}$ & 14 & 13 & 17 & 17 & 17 & 18 \\
$\mathbf{5}$ & 14 & 12 & 14 & 18 & 14 & 14 \\
$\mathbf{6}$ & 12 & 14 & 15 & 15 & 18 & 18 \\
$\mathbf{7}$ & 10 & 10 & 12 & 15 & 14 & 16 \\
$\mathbf{8}$ & 8 & 8 & 8 & 10 & 11 & 11 \\
$\mathbf{9}$ & 3 & $\mathbf{2}$ & $\mathbf{3}$ & 5 & 7 & 5 \\
$\mathbf{1 0 a}$ & 4 & 3 & 5 & $\mathbf{4}$ & $\mathbf{4}$ & $\mathbf{3}$ \\
$\mathbf{1 0 b}$ & 5 & 3 & $\mathbf{2}$ & 6 & 6 & 8 \\
$\mathbf{1 0 c}$ & $\mathbf{1}$ & $\mathbf{2}$ & $\mathbf{2}$ & $\mathbf{3}$ & $\mathbf{5}$ & $\mathbf{3}$ \\
$\mathbf{1 1 a}$ & 12 & 14 & 15 & 15 & 18 & 18 \\
$\mathbf{1 1 b}$ & 10 & 15 & 17 & 14 & 15 & 15 \\
$\mathbf{1 2 a}$ & 2 & 3 & $\mathbf{3}$ & 5 & 6 & 5 \\
$\mathbf{1 2 b}$ & 5 & 4 & 5 & 8 & 6 & 8 \\
$\mathbf{1 3}$ & 11 & 10 & 10 & 11 & 12 & 16 \\
$\mathbf{1 4}$ & 12 & 13 & 13 & 10 & 12 & 14 \\
$\mathbf{1 5}$ & 3 & 3 & 2 & $\mathbf{3}$ & 4 & $\mathbf{4}$ \\
Norfloxacin & 2 & 2 & 3 & 4 & 5 & 4 \\
\hline & & & & & & \\
\hline
\end{tabular}


Antifungal screening showed that most of the tested compounds possessed antifungal activity against all fungal strains (Table IV). Compound 5 exhibited higher activity aganist $C$. albicans and G.lucidum and was equal to MIC of fluconazole against $A$. flavus This may be is attributed to the presence of an intact thiourea $\mathrm{NH}-(\mathrm{C}=\mathrm{S})-\mathrm{NH}$ grouping attached to the thioxopyrimidine. On the other hand, compounds 8, 9, 10a,b, 12a,b, 14, and 15 revealed good antifungal activity against the examined fungi compared to fluconazole as reference antifungal, while the other compounds $(2,3 \mathbf{a}, \mathbf{b}, 4,6,7,10 c, 11 a, b$ and 13$)$ exhibited moderate antifungal activities.

Table IV. Minimal inhibitory concentration (MIC, $\mu \mathrm{mol} \mathrm{mL}^{-1}$ ) of the synthesized compounds against fungi

\begin{tabular}{cccc}
\hline \multirow{2}{*}{ Compd. } & \multicolumn{3}{c}{ Microorganism } \\
\cline { 2 - 4 } & C. albicans & A. flavus & G. lucidum \\
\hline $\mathbf{2}$ & 27 & 22 & 21 \\
3a & 20 & 17 & 22 \\
3b & 23 & 24 & 21 \\
$\mathbf{4}$ & 21 & 24 & 26 \\
$\mathbf{5}$ & $\mathbf{2}$ & $\mathbf{2}$ & $\mathbf{2}$ \\
$\mathbf{6}$ & 18 & 24 & 22 \\
$\mathbf{7}$ & 17 & 17 & 21 \\
$\mathbf{8}$ & 14 & 12 & 12 \\
$\mathbf{9}$ & 11 & 12 & 14 \\
$\mathbf{1 0 a}$ & 13 & 17 & 14 \\
$\mathbf{1 0 b}$ & 8 & 7 & 8 \\
$\mathbf{1 0 c}$ & 25 & 27 & 22 \\
$\mathbf{1 1 a}$ & 24 & 21 & 23 \\
$\mathbf{1 1 b}$ & 22 & 24 & 18 \\
$\mathbf{1 2 a}$ & 10 & 10 & 14 \\
$\mathbf{1 2 b}$ & 14 & 12 & 14 \\
$\mathbf{1 3}$ & 17 & 20 & 17 \\
$\mathbf{1 4}$ & 7 & 6 & 6 \\
$\mathbf{1 5}$ & 11 & 7 & 14 \\
Fluconazole & 3 & 2 & 3 \\
\hline & & & \\
\hline
\end{tabular}

\section{CONCLUSIONS}

The objective of the present study was to synthesize and investigate the antimicrobial activity of some novel pyrazolopyranopyrimidine, pyrazolopyranotriazine and pyrazolopyranotriazolo- pyrimidine derivatives. Compound 5-(4-fluorophenyl)-6-methyl-4-methylpiperazin-1-yl)-5,8-dihydropyrazolo[4',3':5,6]pyrano[2,3-d][1,2,3]triazine (10c) was found to have the most potent antibacterial activity slightly higher than that of norfloxacine. On 
H. N. Hafez et al.: Facile heterocyclic synthesis and antimicrobial activity of polysubstituted and condensed pyrazolopyranopyrimidine and pyrazolopyranotriazine derivatives, Acta Pharm. 65 (2015) 399-412.

the other hand, compound N-[4-(4-fluoro-phenyl)-3-methyl-7-thioxo-1,4,7,8-tetrahydropyrazolo[4',3':5,6]pyrano[2,3- $d]$-pyrimidine-5-yl]thiourea (5) exhibited higher potency of antifungal activity than fluconazole.

Acknowledgements. - The authors are grateful to the Micro-analytical Unit, Cairo University, Egypt, for micro-analytical data, IR, NMR and mass spectra. The authors are also grateful to the Fermentation Biotechnology and Applied Microbiology (Ferm-BAM) Center, Al-Azhar University, Faculty of Science, Cairo, Egypt. The authors extend their sincere appreciation to the Deanship of Scientific Research at the Al-Imam Mohammad Ibn Saud Islamic University for its funding of this research through the Research Group Project no. 341212.

\section{REFERENCES}

1. A. B. A. El-Gazzar and H. N. Hafez, Synthesis of 4-substituted pyrido[2,3- $d$ ]pyrimidin-4(1H)-one as analgesic and anti-inflammatory agents, Bioorg. Med. Chem. Lett. 19 (2009) 3392-3397; DOI: 10.1016/j.bmcl.2009.05.044.

2. F. M. A. El-Taweel, M. A. Sofan, M. A. Mashaly, M. A. Hanna and A. A. Elagamey, Synthesis of some new pyranoquinoline, pyridine and pyrone derivatives, Pharmazie 45 (1990) 671-673; DOI: 10.1002/chin.199149187.

3. A. M. El-Agrody, M. S. Abd El-Latif, N. A. El- Hady, A. H. Fakery and A. H. Bedair, Heteroaromatization with 4-hydroxycoumarin Part II: Synthesis of some new pyrano[2,3-d]-pyrimidines, $[1,2,4]$ triazolo[1,5-c]pyrimidines and pyrimido[1,6-b][1,2,4]triazine derivatives, Molecules 6 (2001) 519-527; DOI: 10.3390/60600519.

4. A. H. Bedair, N. A. El-Haddy, M. S. Abd El-Latif, A. H. Fakery and A. M. El-Agrody, 4- Hydroxycoumarin in heterocyclic synthesis. Part III: Synthesis of some new pyrano[2,3-d][1,2,4]triazolo[1,5c]pyrimidine and pyrimido[1,6-b][1,2,4]triazine derivatives, Farmaco 55 (2000) 708-714; DOI: 10.1016/S0014-827X(00)00097-5.

5. A. M. El-Agrody, M. H. El-Hakim, M. S. Abd El-Latif, A. H. Fakery, E. M. El-sayed and K. A. ElGhareab, Synthesis of pyrano[2,3-d]pyrimidine and pyrano[3,2-e][1,2,4] triazolo[2,3-c] pyrimidine derivatives with promising antibacterial activities, Acta Pharm. 50 (2000) 111-120.

6. A. G. Martinez and L. J. Marco, Friedlander reaction on 2-amino-3-cyano-4H-pyrans, synthesis of derivatives of 4H-pyran[2,3-b]quinoline, new tacrine analogues, Bioorg. Med. Chem. Lett. 7 (1997) 3165-3170; DOI: 10.1016/S0960-894X(97)10165-2.

7. F. Eiden and F. Denk, Synthesis and CNS activity of pyrane derivatives: 6,8-dioxabicyclo(3,2,1) octanes, Arch. Pharm. (Weinheim) 324 (1991) 353-354.

8. C. P. Dell and C. W. Smith, Antiproliferative derivatives of $4 H$-naphtho[1,2-b]pyran and process for their preparation, Eur. Pat. 537, 94, 921 Apr 1993; ref. Chem. Abstr. 119 (1993) 139102d.

9. F. M. Abdelrazek, P. Metz, N. H. Metwally and S. F. El-Mahrouky, Synthesis and molluscicidal activity of new cinnoline and pyrano[2,3-c]pyrazole derivatives, Arch. Pharm. Chem. Life Sci. 339 (2006) 456-460.

10. G. Bianchi and A. Tava, Synthesis of $(2 R)(+)-2,3$-dihydro-2,6-dimethyl-4H-pyran-4-one, a homologue of pheromones of a species in the hepialidae family, Agr. Biol. Chem. 51 (1987) 2001-2002; DOI: 10.1271/bbb1961.51.2001.

11. K. Noda, A. Nakagawa, Y. Nakajima and H. Ide, Pyrido[2,3- $d$ ]-s-triazolo[4,3-c]pyrimidine derivatives, Japan Kokai 7785, 194, 15 Jul 1977; ref. Chem. Abstr. 88 (1978) P50908q. 
H. N. Hafez et al.: Facile heterocyclic synthesis and antimicrobial activity of polysubstituted and condensed pyrazolopyranopyrimidine and pyrazolopyranotriazine derivatives, Acta Pharm. 65 (2015) 399-412.

12. H. N. Hafez, H. A. S. Abbas and A. B. A. El-Gazzar, Synthesis and evaluation of analgesic, antiinflammatory and ulcerogenic activities of some triazolo- and 2-pyrazolyl-pyrido[2,3-d]-pyrimidines, Acta. Pharm. 58 (2008) 359-378; DOI: 10.2478/v10007-008-0024-1.

13. A. B. A. El-Gazzar, H. A. R. Hossein and H. N. Hafez, Synthesis and biological evaluation of thieno[2,3-d]pyrimidines derivatives as anti-inflammatory, analgesic and ulcerogenic activity, Acta. Pharm. 57 (2007) 395-411; DOI: 10.2478/v10007-007-0032-6.

14. A. B. A. El-Gazzar, H. N. Hafez and G. M. Nawwar, New acyclic nucleosides analogues as potential analgesic, anti-inflammatory, anti-oxidant and anti-microbial derived from pyrimido[4,5- $b]$ quinolines, Eur. Med. Chem. 44 (2009) 1427-1436; DOI: 10.1016/j.ejmech. 2008.09.030.

15. L. P. Prikazchikova, B. M. Khutova, I. F. Vladimirtsev, I. V. Boldyrev and N. I. Zhuravskaya, stimulating activity of mono- and diketone pyrimidine derivatives, Fiziol. Akt. Veshchestva 7 (1975) 84-87; ref. Chem. Abstr. 83 (1975) 127346m.

16. D. J. Brown, Pyrimidines and Their Benzo Derivatives, in Comprehensive Heterocyclic Chemistry (Eds. A. R. Katritzky and C. W. Rees), Vol. 3, Pergamon Press, Oxford 1984, p. 443.

17. S. M. Yu, S. C. Kuo, L. J. Huang, S. S. M. Sun, T. F. Huang and C. M. Teng, Vasorelaxation of rat thoracic aorta caused by two $\mathrm{Ca}^{2+}$-channel blockers, HA-22 and HA-23, J. Pharm. Pharmacol. 44 (1992) 667-671; DOI: 10.1111/j.2042-7158.1992.tb05491.x.

18. D. Catarzi, L. Cecchi, V. Colotta, G. Filacchioni, C. Martini, P. Tacchi and A. Lucacchini, Tricyclic heteroaromatic systems. Synthesis and A1 and A2a adenosine binding activities of some 1-aryl1,4-dihydro-3-methyl[1]benzopyrano[2,3-c]pyrazol-4-ones, 1-aryl-4,9-dihydro-3-methyl-1Hpyrazolo[3,4-b]quinolin-4-ones, and 1-aryl-1H-imidazo[4,5-b]quinoxalines, J. Med. Chem. 38 (1995) 1330-1336.

19. N. Foloppe, L. M. Fisher, R. Howes, A. Potter, A. G. S. Robertson and A. E. Surgenor, Identification of chemically diverse Chk1 inhibitors by receptor-based virtual screening, Bioorg. Med. Chem. 14 (14) (2006) 4792-4802; DOI: 10.1016/j.bmc.2006.03.021.

20. H. M. Al-Matar, K. D. Khalil, A. Y. Adam and M. H. Elnagdi, Green one pot solvent-free synthesis of pyrano[2,3-c]pyrazoles and pyrazolo[1,5-a]pyrimidines, Molecules 15 (2010) 6619-6629; DOI: 10.3390/molecules15096619.

21. S. R. Jain and A. Kar, The antibacterial activity of some essential oils and their combination, Planta Med. 20 (1971) 118-123; DOI: 10.1055/s-0028-109967.

22. H. Naeimi, Z. S. Nazifi, S. M. Amininezhad and M. Amouheidari, Synthesis, characterization and in vitro antimicrobial activity of some new Schiff bases and their complexes, J. Antibiot. 66 (2013) 687-689; DOI: 10.1038/ja.2013.73.

23. M. B. Madhusudana Reddy, V. P. Jayashankara and M. A. Pasha, Glycine-catalyzed efficient synthesis of pyranopyrazoles via one-pot multicomponent reaction, Synth. Commun. 40 (2010) 29302934; DOI: 10.1080/00397910903340686.

24. D. Thomae, G. Kirsch and P. Seck, Synthesis of selenophene analogues of the tacrine series: comparison of classical route and microwave irradiation, Synthesis 10 (2008) 1600-1606; DOI: 10.1055/s2008-1067001.

25. N. Galic, B. Peric, B. Kojic-Prodic and Z. Cimerman, Structural and spectroscopic characteristics of aroylhydrazones derived from nicotinic acid hydrazide, J. Mol. Struct. 559 (2001) 187-194; DOI: 10.10.1016/S0022-2860(00)00703-1.

26. V. Klimesova, L. Zahajska, K. Waisser, J. Kaustova and U. Mollmann, Synthesis and antimycobacterial activity of 1, 2, 4-triazole 3-benzylsulfanyl derivatives, Farmaco 59 (2004) 279-288; DOI: 10.1016/j.farmac.2004.01.006. 